\title{
Sherman-Morrison-Woodbury Formula for Linear Integrodifferential Equations
}

\begin{abstract}
Feng Wu
State Key Laboratory of Structural Analysis of Industrial Equipment, Faculty of Vehicle Engineering and Mechanics, Dalian University of Technology, Dalian 116023, China

Correspondence should be addressed to Feng Wu; wufeng_chn@163.com

Received 22 September 2016; Accepted 4 October 2016

Academic Editor: Kishin Sadarangani

Copyright (c) 2016 Feng Wu. This is an open access article distributed under the Creative Commons Attribution License, which permits unrestricted use, distribution, and reproduction in any medium, provided the original work is properly cited.

The well-known Sherman-Morrison-Woodbury formula is a powerful device for calculating the inverse of a square matrix. The paper finds that the Sherman-Morrison-Woodbury formula can be extended to the linear integrodifferential equation, which results in an unified scheme to decompose the linear integrodifferential equation into sets of differential equations and one integral equation. Two examples are presented to illustrate the Sherman-Morrison-Woodbury formula for the linear integrodifferential equation.
\end{abstract}

\section{Introduction}

This paper is devoted to the study of the linear integrodifferential equation (IDE):

$$
\begin{gathered}
L(v(\boldsymbol{\theta}, \mathbf{x}))+k(\boldsymbol{\theta}, \mathbf{x}) \int_{\Theta(\boldsymbol{\theta})} Q(\boldsymbol{\theta}, \mathbf{x}, \mathbf{s}, D(v(\mathbf{s}, \mathbf{x}))) \mathrm{d} \mathbf{s} \\
\quad=f(\boldsymbol{\theta}, \mathbf{x}), \quad \mathbf{x} \in \Omega, \boldsymbol{\theta} \in \Theta, \Theta(\boldsymbol{\theta}) \subseteq \Theta
\end{gathered}
$$

with the homogeneous boundary conditions:

$$
\begin{aligned}
& B(v(\boldsymbol{\theta}, \mathbf{x}))=0, \quad \mathbf{x} \in \partial \Omega, \\
& C(v(\boldsymbol{\theta}, \mathbf{x}))=0, \quad \boldsymbol{\theta} \in \partial \Theta,
\end{aligned}
$$

where

$$
\begin{aligned}
& \boldsymbol{\theta}=\left(\begin{array}{llll}
\theta_{1} & \theta_{2} & \cdots & \theta_{m}
\end{array}\right)^{\mathrm{T}} \in \Theta, \\
& \mathbf{x}=\left(\begin{array}{llll}
x_{1} & x_{2} & \cdots & x_{n}
\end{array}\right)^{\mathrm{T}} \in \Omega,
\end{aligned}
$$

$L(v(\boldsymbol{\theta}, \mathbf{x})), B(v(\boldsymbol{\theta}, \mathbf{x})), C(v(\boldsymbol{\theta}, \mathbf{x}))$, and $D(v(\boldsymbol{\theta}, \mathbf{x}))$ are all linear differential operators. $Q(\boldsymbol{\theta}, \mathbf{s}, D(v(\mathbf{s}, \mathbf{x})))$ is a linear function with respect to $D(v(\boldsymbol{\theta}, \mathbf{x})) . \Theta(\boldsymbol{\theta}) \subseteq \Theta$ is dependent on the variable $\boldsymbol{\theta}, v(\boldsymbol{\theta}, \mathbf{x})$ is the unknown function of two types of variables, that is, $\boldsymbol{\theta}$ and $\mathbf{x}$. However, the integration in (1) is with respect to $\boldsymbol{\theta}$, without involving $\mathbf{x}$.
The IDE can be found to describe various kinds of phenomena such as Bose-Einstein condensates [1], wavepower hydraulics [2], heterogeneous heat transfer [3], slow erosion of granular flow [4], and wind ripple in the desert [5]. Solving this equation has been one of the interesting tasks for researchers. To date, there are many numerical methods such as finite element method [6] and finite difference method [7] that can be used to obtain an approximate solution for this problem. There are also some analytical methods such as the classical polynomial expansion method [8], the operational approach [9], the homotopy perturbation method [10], the separation of variables technique [11], and the resolvent method [12]. When we employ these analytical methods to solve the IDE, one common feature is that the IDE is converted to a series of pure differential equations and pure integral equations. In this article we propose a new technique for (1), that is, the Sherman-Morrison-Woodbury (SMW) formula [13]. The SMW formula is a powerful device for calculating the inverse matrix of a square matrix, which will be described briefly in Section 2. In Section 3, we extend the SMW formula to solve the IDE. We find that the SMW formula provides a unified procedure to decompose the linear IDE into several differential equations and one pure integral equation. In Section 4 two simple examples are used to explain this scheme. 


\section{Sherman-Morrison-Woodbury Formula}

The SMW formula was developed for calculating the inverse matrix of a square matrix. Suppose that we have already obtained, by herculean effort, the inverse matrix $\mathbf{A}^{-1}$ of a square matrix $\mathbf{A}$. Then a small change, for example, $\Delta \mathbf{A}=$ $\mathbf{a b}^{\mathrm{T}}$, in $\mathbf{A}$ happens, and we need to calculate the inverse matrix $(\mathbf{A}+\Delta \mathbf{A})^{-1}$. SMW formula is a powerful device for this problem.

Lemma 1 (Sherman-Morrison-Woodbury formula). If $\Delta \mathbf{A}=$ $\mathbf{a b}^{\mathrm{T}}$, the inverse matrix of $\mathbf{A}+\Delta \mathbf{A}$ is

$$
(\mathbf{A}+\Delta \mathbf{A})^{-1}=\mathbf{A}^{-1}-\mathbf{A}^{-1} \mathbf{a}\left(\mathbf{I}+\mathbf{b}^{\mathrm{T}} \mathbf{A}^{-1} \mathbf{a}\right)^{-1} \mathbf{b}^{\mathrm{T}} \mathbf{A}^{-1} .
$$

Proof. Refer to [13].

It can be observed from (4) that calculating $(\mathbf{A}+\Delta \mathbf{A})^{-1}$ is converted to calculating $\left(\mathbf{I}+\mathbf{b}^{\mathrm{T}} \mathbf{A}^{-1} \mathbf{a}\right)^{-1}$. In the next section we will develop the IDE version of the SMW formula.

\section{SMW Formula for the IDE}

In this section we extend the SMW formula to the IDE.

Definition 2. The function $w(\mathbf{s}, \mathbf{c} ; \boldsymbol{\theta}, \mathbf{x})$ is the Green function of the following differential equation (5), if it satisfies

$$
\begin{aligned}
& L(w(\mathbf{s}, \mathbf{c} ; \boldsymbol{\theta}, \mathbf{x}))=\delta(\mathbf{x}-\mathbf{c}) \delta(\boldsymbol{\theta}-\mathbf{s}), \quad \mathbf{x} \in \Omega, \boldsymbol{\theta} \in \Theta, \\
& B(w(\mathbf{s}, \mathbf{c} ; \boldsymbol{\theta}, \mathbf{x}))=0, \quad \mathbf{x} \in \partial \Omega, \\
& C(w(\mathbf{s}, \mathbf{c} ; \boldsymbol{\theta}, \mathbf{x}))=0, \quad \boldsymbol{\theta} \in \partial \Theta,
\end{aligned}
$$

where

$$
\begin{aligned}
\mathbf{s} & =\left(\begin{array}{llll}
s_{1} & s_{2} & \cdots & s_{m}
\end{array}\right)^{\mathrm{T}} \in \Theta, \\
\mathbf{c} & =\left(\begin{array}{llll}
c_{1} & c_{2} & \cdots & c_{n}
\end{array}\right)^{\mathrm{T}} \in \Omega, \\
\delta(\mathbf{x}-\mathbf{c}) & =\prod_{i=1}^{n} \delta\left(x_{i}-c_{i}\right), \\
\delta(\boldsymbol{\theta}-\mathbf{s}) & =\prod_{j=1}^{m} \delta\left(\theta_{i}-s_{i}\right) .
\end{aligned}
$$

its solution is

$$
u(\boldsymbol{\theta}, \mathbf{x})=\int_{\Theta} \int_{\Omega} w(\mathbf{s}, \mathbf{c} ; \boldsymbol{\theta}, \mathbf{x}) f(\mathbf{s}, \mathbf{c}) \mathrm{d} \mathbf{s} \mathrm{d} \mathbf{c} .
$$

Proof. Substituting (8) into (7), we have

$$
\begin{aligned}
L(u(\boldsymbol{\theta}, \mathbf{x})) & =L\left(\int_{\Theta} \int_{\Omega} w(\mathbf{s}, \mathbf{c} ; \boldsymbol{\theta}, \mathbf{x}) f(\mathbf{s}, \mathbf{c}) \mathrm{d} \mathbf{s} \mathrm{d} \mathbf{c}\right) \\
& =\int_{\Theta} \int_{\Omega} \delta(\mathbf{x}-\mathbf{c}) \delta(\boldsymbol{\theta}-\mathbf{s}) f(\mathbf{s}, \mathbf{c}) \mathrm{d} \mathbf{s} \mathrm{d} \mathbf{c} \\
& =f(\boldsymbol{\theta}, \mathbf{x}), \\
B(u(\boldsymbol{\theta}, \mathbf{x})) & =B\left(\int_{\Theta} \int_{\Omega} w(\mathbf{s}, \mathbf{c} ; \boldsymbol{\theta}, \mathbf{x}) f(\mathbf{s}, \mathbf{c}) \mathrm{d} \mathbf{s} \mathrm{d} \mathbf{c}\right) \\
& =\int_{\Theta} \int_{\Omega} B(w(\mathbf{s}, \mathbf{c} ; \boldsymbol{\theta}, \mathbf{x})) f(\mathbf{s}, \mathbf{c}) \mathrm{d} \mathbf{s} \mathrm{d} \mathbf{c}=0, \\
C(u(\boldsymbol{\theta}, \mathbf{x})) & =C\left(\int_{\Theta} \int_{\Omega} w(\mathbf{s}, \mathbf{c} ; \boldsymbol{\theta}, \mathbf{x}) f(\mathbf{s}, \mathbf{c}) \mathrm{d} \mathbf{s} \mathrm{d} \mathbf{c}\right) \\
& =\int_{\Theta} \int_{\Omega} C(w(\mathbf{s}, \mathbf{c} ; \boldsymbol{\theta}, \mathbf{x})) f(\mathbf{s}, \mathbf{c}) \mathrm{d} \mathbf{s} \mathrm{d} \mathbf{c}=0 .
\end{aligned}
$$

So the proof of the lemma is completed.

Note that in the proof we assume that the linear differential operators $L, B$, and $C$ and the integral operator $\int_{\Theta} \int_{\Omega}(\cdot)$ are commutative. The assumption holds throughout the paper.

Theorem 4. The solution of (5) can be written as

$$
v(\boldsymbol{\theta}, \mathbf{x})=u(\boldsymbol{\theta}, \mathbf{x})-p(\boldsymbol{\theta}, \mathbf{x}),
$$

where $u(\boldsymbol{\theta}, \mathbf{x})$ satisfies

$$
\begin{aligned}
& L(u(\boldsymbol{\theta}, \mathbf{x}))=f(\boldsymbol{\theta}, \mathbf{x}), \quad \mathbf{x} \in \Omega, \boldsymbol{\theta} \in \Theta, \\
& B(u(\boldsymbol{\theta}, \mathbf{x}))=0, \quad \mathbf{x} \in \partial \Omega, \\
& C(u(\boldsymbol{\theta}, \mathbf{x}))=0, \quad \boldsymbol{\theta} \in \partial \Theta,
\end{aligned}
$$

$p(\boldsymbol{\theta}, \mathbf{x})$ satisfies

$$
\begin{aligned}
& L(p(\boldsymbol{\theta}, \mathbf{x}))=k(\boldsymbol{\theta}, \mathbf{x}) g(\boldsymbol{\theta}, \mathbf{x}), \quad \mathbf{x} \in \Omega, \boldsymbol{\theta} \in \Theta, \\
& B(p(\boldsymbol{\theta}, \mathbf{x}))=0, \quad \mathbf{x} \in \partial \Omega, \\
& C(p(\boldsymbol{\theta}, \mathbf{x}))=0, \quad \boldsymbol{\theta} \in \partial \Theta,
\end{aligned}
$$

and $g(\boldsymbol{\theta}, \mathbf{x})$ satisfies

$$
\begin{gathered}
g(\boldsymbol{\theta}, \mathbf{x})+\int_{\Theta} \int_{\Omega} q(\mathbf{s}, \mathbf{c} ; \boldsymbol{\theta}, \mathbf{x}) k(\mathbf{s}, \mathbf{c}) g(\mathbf{s}, \mathbf{c}) \mathrm{d} \mathbf{s} \mathrm{d} \mathbf{c} \\
=\int_{\Theta(\boldsymbol{\theta})} Q(\boldsymbol{\theta}, \mathbf{x}, \mathbf{s}, D(u(\mathbf{s}, \mathbf{x}))) \mathrm{d} \mathbf{s}
\end{gathered}
$$

in which

$$
q(\mathbf{s}, \mathbf{c} ; \boldsymbol{\theta}, \mathbf{x})=\int_{\Theta(\boldsymbol{\theta})} Q(\boldsymbol{\theta}, \mathbf{x}, \boldsymbol{\alpha},(D(w(\mathbf{s}, \mathbf{c} ; \boldsymbol{\alpha}, \mathbf{x})))) \mathrm{d} \boldsymbol{\alpha} .
$$

Proof. It can be observed easily in terms of (11) and (12) that (10) satisfies the boundary conditions defined by (2). 
Substituting $u(\boldsymbol{\theta}, \mathbf{x})-p(\boldsymbol{\theta}, \mathbf{x})$ into the left side of (1) and using (11) and (12), we have

$$
\begin{aligned}
& L(v(\boldsymbol{\theta}, \mathbf{x}))+k(\boldsymbol{\theta}, \mathbf{x}) \int_{\Theta(\boldsymbol{\theta})} \mathrm{Q}(\boldsymbol{\theta}, \mathbf{x}, \mathbf{s}, D(v(\mathbf{s}, \mathbf{x}))) \mathrm{d} \mathbf{s} \\
& =L(u(\boldsymbol{\theta}, \mathbf{x}))-L(p(\boldsymbol{\theta}, \mathbf{x})) \\
& \quad+k(\boldsymbol{\theta}, \mathbf{x}) \int_{\Theta(\boldsymbol{\theta})} Q(\boldsymbol{\theta}, \mathbf{x}, \mathbf{s}, D(u(\mathbf{s}, \mathbf{x}))) \mathrm{d} \mathbf{s} \\
& \quad-k(\boldsymbol{\theta}, \mathbf{x}) \int_{\Theta(\boldsymbol{\theta})} Q(\boldsymbol{\theta}, \mathbf{x}, \mathbf{s}, D(p(\mathbf{s}, \mathbf{x}))) \mathrm{d} \mathbf{s} \\
& =f(\boldsymbol{\theta}, \mathbf{x})-k(\boldsymbol{\theta}, \mathbf{x}) g(\boldsymbol{\theta}, \mathbf{x}) \\
& \quad+k(\boldsymbol{\theta}, \mathbf{x}) \int_{\Theta(\boldsymbol{\theta})} Q(\boldsymbol{\theta}, \mathbf{x}, \mathbf{s}, D(u(\mathbf{s}, \mathbf{x}))) \mathrm{d} \mathbf{s} \\
& \quad-k(\boldsymbol{\theta}, \mathbf{x}) \int_{\Theta(\boldsymbol{\theta})} Q(\boldsymbol{\theta}, \mathbf{x}, \mathbf{s}, D(p(\mathbf{s}, \mathbf{x}))) \mathrm{d} \mathbf{s} .
\end{aligned}
$$

In terms of Lemma 3, the solution of (12) can be written as

$$
p(\mathbf{s}, \mathbf{x})=\int_{\Theta} \int_{\Omega} w(\boldsymbol{\alpha}, \mathbf{c} ; \mathbf{s}, \mathbf{x}) k(\boldsymbol{\alpha}, \mathbf{c}) g(\boldsymbol{\alpha}, \mathbf{c}) \mathrm{d} \boldsymbol{\alpha} \mathrm{d} \mathbf{c} .
$$

Using (14) and (16) to the last term in the right side of (15) yields

$$
\begin{aligned}
& \int_{\Theta(\boldsymbol{\theta})} Q(\boldsymbol{\theta}, \mathbf{x}, \mathbf{s}, D(p(\mathbf{s}, \mathbf{x}))) \mathrm{d} \mathbf{s}=\int_{\Theta(\boldsymbol{\theta})} Q(\boldsymbol{\theta}, \mathbf{x}, \mathbf{s}, \\
& \left.D\left(\int_{\Theta} \int_{\Omega} w(\boldsymbol{\alpha}, \mathbf{c} ; \mathbf{s}, \mathbf{x}) k(\boldsymbol{\alpha}, \mathbf{c}) g(\boldsymbol{\alpha}, \mathbf{c}) \mathrm{d} \boldsymbol{\alpha} \mathrm{d} \mathbf{c}\right)\right) \mathrm{d} \mathbf{s} \\
& =\int_{\Theta} \int_{\Omega}\left[\int_{\Theta(\boldsymbol{\theta})} Q(\boldsymbol{\theta}, \mathbf{x}, \mathbf{s}, D(w(\boldsymbol{\alpha}, \mathbf{c} ; \mathbf{s}, \mathbf{x}))) \mathrm{d} \mathbf{s}\right] \\
& \cdot k(\boldsymbol{\alpha}, \mathbf{c}) g(\boldsymbol{\alpha}, \mathbf{c}) \mathrm{d} \boldsymbol{\alpha} \mathrm{d} \mathbf{c}=\int_{\Theta} \int_{\Omega} q(\boldsymbol{\alpha}, \mathbf{c} ; \boldsymbol{\theta}, \mathbf{x}) \\
& \cdot k(\boldsymbol{\alpha}, \mathbf{c}) g(\boldsymbol{\alpha}, \mathbf{c}) \mathrm{d} \boldsymbol{\alpha} \mathrm{d} \mathbf{c}=\int_{\Theta} \int_{\Omega} q(\mathbf{s}, \mathbf{c} ; \boldsymbol{\theta}, \mathbf{x}) \\
& \cdot k(\boldsymbol{\alpha}, \mathbf{c}) g(\mathbf{s}, \mathbf{c}) \mathrm{d} \mathbf{s} \mathrm{d} \mathbf{c} .
\end{aligned}
$$

Substitute (17) into (15) and then use (29) to the result

$$
\begin{aligned}
& L(v(\boldsymbol{\theta}, \mathbf{x}))+k(\boldsymbol{\theta}, \mathbf{x}) \int_{\Theta(\boldsymbol{\theta})} \mathrm{Q}(\boldsymbol{\theta}, \mathbf{x}, \mathbf{s}, D(v(\mathbf{s}, \mathbf{x}))) \mathrm{d} \mathbf{s} \\
& \quad=L(u(\boldsymbol{\theta}, \mathbf{x}))-L(p(\boldsymbol{\theta}, \mathbf{x}))+k(\boldsymbol{\theta}, \mathbf{x}) \\
& \quad \cdot \int_{\Theta(\boldsymbol{\theta})} Q(\boldsymbol{\theta}, \mathbf{x}, \mathbf{s}, D(u(\mathbf{s}, \mathbf{x}))) \mathrm{d} \mathbf{s}-k(\boldsymbol{\theta}, \mathbf{x}) \\
& \quad \cdot \int_{\Theta(\boldsymbol{\theta})} Q(\boldsymbol{\theta}, \mathbf{x}, \mathbf{s}, D(p(\mathbf{s}, \mathbf{x}))) \mathrm{d} \mathbf{s}=f(\boldsymbol{\theta}, \mathbf{x})
\end{aligned}
$$

$$
\begin{aligned}
& -k(\boldsymbol{\theta}, \mathbf{x})[g(\boldsymbol{\theta}, \mathbf{x}) \\
& +\int_{\Theta} \int_{\Omega} q(\mathbf{s}, \mathbf{c} ; \boldsymbol{\theta}, \mathbf{x}) k(\mathbf{s}, \mathbf{c}) g(\mathbf{s}, \mathbf{c}) \mathrm{d} \mathbf{s} \mathrm{d} \mathbf{c} \\
& \left.-\int_{\Theta(\boldsymbol{\theta})} Q(\boldsymbol{\theta}, \mathbf{x}, \mathbf{s}, D(u(\mathbf{s}, \mathbf{x}))) \mathrm{d} \mathbf{s}\right]=f(\boldsymbol{\theta}, \mathbf{x}) .
\end{aligned}
$$

So the proof of the theorem is completed.

Note that in the proof we assume that the linear differential operators $Q, D$ and the integral operator $\int_{\Theta} \int_{\Omega}(\cdot)$ are commutative. The assumption holds throughout the paper.

Equation (10) can be seen as the IDE version of the Sherman-Morrison-Woodbury formula. In order to explain this point more clearly, we introduce some notations.

Definition 5. The following linear operators are defined by

$$
\begin{aligned}
& L \circ v(\boldsymbol{\theta}, \mathbf{x})=L(v(\boldsymbol{\theta}, \mathbf{x})), \\
& S \circ v(\boldsymbol{\theta}, \mathbf{x})=\int_{\Theta(\boldsymbol{\theta})} Q(\boldsymbol{\theta}, \mathbf{x}, \mathbf{s}, D(v(\mathbf{s}, \mathbf{x}))) \mathrm{d} \mathbf{s} .
\end{aligned}
$$

Definition 6. The inverse linear operator of $L$ is defined by

$$
\begin{aligned}
L^{-1} \circ f(\mathbf{x}, \boldsymbol{\theta}) & =u(\mathbf{x}, \boldsymbol{\theta}) \\
& =\int_{\Theta} \int_{\Omega} w(\mathbf{s}, \mathbf{c} ; \boldsymbol{\theta}, \mathbf{x}) f(\mathbf{s}, \mathbf{c}) \mathrm{d} \mathbf{s} \mathrm{d} \mathbf{c},
\end{aligned}
$$

where $w(\mathbf{s}, \mathbf{c} ; \boldsymbol{\theta}, \mathbf{x})$ is the Green function defined by (5).

Using (19) and (20) to (1) yields

$$
\begin{aligned}
L(v(\boldsymbol{\theta}, \mathbf{x}))+k(\boldsymbol{\theta}, \mathbf{x}) \cdot S \circ v(\boldsymbol{\theta}, \mathbf{x})=f(\boldsymbol{\theta}, \mathbf{x}), & \\
& \mathbf{x} \in \Omega, \boldsymbol{\theta} \in \Theta .
\end{aligned}
$$

Lemma 7. Equation (29) can be rewritten as

$$
g(\boldsymbol{\theta}, \mathbf{x})+S \circ L^{-1} \circ k(\boldsymbol{\theta}, \mathbf{x}) \cdot g(\boldsymbol{\theta}, \mathbf{x})=S \circ L^{-1} f(\boldsymbol{\theta}, \mathbf{x}) .
$$

Proof. In terms of (19), we have

$$
\begin{aligned}
& \int_{\Omega} \int_{\Theta} q(\mathbf{s}, \mathbf{c} ; \boldsymbol{\theta}, \mathbf{x}) f(\mathbf{s}, \mathbf{c}) \mathrm{d} \mathbf{s} \mathrm{d} \mathbf{c} \\
& \quad=\int_{\Omega} \int_{\Theta} \int_{\Theta(\boldsymbol{\theta})} Q(\boldsymbol{\theta}, \mathbf{x}, \boldsymbol{\alpha},(D(w(\mathbf{s}, \mathbf{c} ; \boldsymbol{\alpha}, \mathbf{x})))) \mathrm{d} \boldsymbol{\alpha} \\
& \quad \cdot f(\mathbf{s}, \mathbf{c}) \mathrm{d} \mathbf{s} \mathrm{d} \mathbf{c}=\int_{\Theta(\boldsymbol{\theta})} Q(\boldsymbol{\theta}, \mathbf{x}, \boldsymbol{\alpha}, \\
& \left.D\left(\int_{\Omega} \int_{\Theta} w(\mathbf{s}, \mathbf{c} ; \boldsymbol{\alpha}, \mathbf{x}) f(\mathbf{s}, \mathbf{c}) \mathrm{d} \mathbf{s} \mathrm{d} \mathbf{c}\right)\right) \mathrm{d} \boldsymbol{\alpha} \\
& =\int_{\Theta(\boldsymbol{\theta})} Q\left(\boldsymbol{\theta}, \mathbf{x}, \boldsymbol{\alpha}, D\left(L^{-1} f(\mathbf{x}, \boldsymbol{\theta})\right)\right) \mathrm{d} \boldsymbol{\alpha}=S \\
& \circ L^{-1} \circ f(\mathbf{x}, \boldsymbol{\theta}),
\end{aligned}
$$

Substituting (23) into (5) yields (22). 
We denote the solution $g(\boldsymbol{\theta}, \mathbf{x})$ of (22) by

$$
g(\boldsymbol{\theta}, \mathbf{x})=\left[1+S \circ L^{-1} \circ k(\boldsymbol{\theta}, \mathbf{x})\right]^{-1} S \circ L^{-1} f(\boldsymbol{\theta}, \mathbf{x}) .
$$

Theorem 8 (Sherman-Morrison-Woodbury formula). The solution of (1) can be written as

$$
\begin{aligned}
v(\boldsymbol{\theta}, \mathbf{x})= & L^{-1} f(\boldsymbol{\theta}, \mathbf{x}) \\
& -L^{-1} k(\boldsymbol{\theta}, \mathbf{x})\left[1+S \circ L^{-1} \circ k(\boldsymbol{\theta}, \mathbf{x})\right]^{-1} S \\
& \circ L^{-1} f(\boldsymbol{\theta}, \mathbf{x}) .
\end{aligned}
$$

Proof. In terms of (20), we have

$$
\begin{aligned}
& u(\boldsymbol{\theta}, \mathbf{x})=L^{-1} \circ f(\boldsymbol{\theta}, \mathbf{x}), \\
& p(\boldsymbol{\theta}, \mathbf{x})=L^{-1} \circ(k(\boldsymbol{\theta}, \mathbf{x}) \cdot g(\boldsymbol{\theta}, \mathbf{x})) .
\end{aligned}
$$

Combining (26) with (24) gives

$$
\begin{aligned}
v(\boldsymbol{\theta}, \mathbf{x})= & u(\boldsymbol{\theta}, \mathbf{x})-p(\boldsymbol{\theta}, \mathbf{x}) \\
= & L^{-1} f(\boldsymbol{\theta}, \mathbf{x})-L^{-1} k(\boldsymbol{\theta}, \mathbf{x}) g(\boldsymbol{\theta}, \mathbf{x}) \\
= & L^{-1} f(\boldsymbol{\theta}, \mathbf{x}) \\
& -L^{-1} k(\boldsymbol{\theta}, \mathbf{x})\left[1+S \circ L^{-1} \circ k(\boldsymbol{\theta}, \mathbf{x})\right]^{-1} S \\
& \circ L^{-1} f(\boldsymbol{\theta}, \mathbf{x}) .
\end{aligned}
$$

So the proof of the theorem is completed.

It can be observed clearly that (25) is the SMW formula for the IDE. Theorems 4 and 8 show that solving the IDE (1) could be converted to solving the integral equation (13) or (29).

According to Lemma 3, the solution of (11) can be written as

$$
u(\mathbf{s}, \mathbf{x})=\int_{\Theta} \int_{\Omega} w(\boldsymbol{\alpha}, \mathbf{c} ; \mathbf{s}, \mathbf{x}) f(\boldsymbol{\alpha}, \mathbf{c}) \mathrm{d} \boldsymbol{\alpha} \mathrm{d} \mathbf{c} .
$$

Using (28) to the right-hand side of (13) yields

$$
\begin{gathered}
g(\boldsymbol{\theta}, \mathbf{x})+\int_{\Theta} \int_{\Omega} q(\mathbf{s}, \mathbf{c} ; \boldsymbol{\theta}, \mathbf{x}) k(\mathbf{s}, \mathbf{c}) g(\mathbf{s}, \mathbf{c}) \mathrm{d} \mathbf{s} \mathrm{d} \mathbf{c} \\
\quad=\int_{\Omega} \int_{\Theta} q(\mathbf{s}, \mathbf{c} ; \boldsymbol{\theta}, \mathbf{x}) f(\mathbf{s}, \mathbf{c}) \mathrm{d} \mathbf{s} \mathrm{d} \mathbf{c} .
\end{gathered}
$$

Multiply both sides of (29) by $k(\boldsymbol{\theta}, \mathbf{x})$ and rearrange the equation:

$$
\begin{aligned}
& k(\boldsymbol{\theta}, \mathbf{x}) g(\boldsymbol{\theta}, \mathbf{x}) \\
& \quad+\int_{\Theta} \int_{\Omega} q(\mathbf{s}, \mathbf{c} ; \boldsymbol{\theta}, \mathbf{x})[k(\mathbf{s}, \mathbf{c}) g(\mathbf{s}, \mathbf{c})-f(\mathbf{s}, \mathbf{c})] \mathrm{d} \mathbf{s} \mathrm{d} \mathbf{c} \\
& \quad=0 .
\end{aligned}
$$

Let

$$
h(\boldsymbol{\theta}, \mathbf{x})=f(\boldsymbol{\theta}, \mathbf{x})-k(\boldsymbol{\theta}, \mathbf{x}) g(\boldsymbol{\theta}, \mathbf{x})
$$

then (30) can be rewritten as

$$
h(\boldsymbol{\theta}, \mathbf{x})+\int_{\Theta} \int_{\Omega} q(\mathbf{s}, \mathbf{c} ; \boldsymbol{\theta}, \mathbf{x}) h(\mathbf{s}, \mathbf{c}) \mathrm{d} \mathbf{s} \mathrm{d} \mathbf{c}=f(\mathbf{s}, \mathbf{c}) .
$$

Noting (10)-(12) and (31), we have the following corollary.

Corollary 9. The solution of (1) satisfies

$$
\begin{aligned}
& L(v(\boldsymbol{\theta}, \mathbf{x}))=h(\boldsymbol{\theta}, \mathbf{x}), \quad \mathbf{x} \in \Omega, \boldsymbol{\theta} \in \Theta, \\
& B(v(\boldsymbol{\theta}, \mathbf{x}))=0, \quad \mathbf{x} \in \partial \Omega, \\
& C(v(\boldsymbol{\theta}, \mathbf{x}))=0, \quad \boldsymbol{\theta} \in \partial \Theta
\end{aligned}
$$

where $h(\boldsymbol{\theta}, \mathbf{x})$ is defined by (32).

In terms of Theorem 4, we can obtain the following corollary.

Corollary 10. If $k(\boldsymbol{\theta}, \mathbf{x})=k(\mathbf{x})$ is independent of the variable $\boldsymbol{\theta}$ and $\mathrm{Q}(\boldsymbol{\theta}, \mathbf{x}, \mathbf{s}, D(v(\mathbf{s}, \mathbf{x})))=\mathrm{Q}(\mathbf{x}, \mathbf{s}, D(v(\mathbf{s}, \mathbf{x})))$ is also independent of the variable $\boldsymbol{\theta}$, then the solution is

$$
v(\boldsymbol{\theta}, \mathbf{x})=L^{-1} \circ f(\boldsymbol{\theta}, \mathbf{x})-L^{-1} \circ[k(\mathbf{x}) \cdot g(\mathbf{x})],
$$

where $g(\mathbf{x})$ satisfies

$$
\begin{gathered}
g(\mathbf{x})+\int_{\Theta} \int_{\Omega} q(\mathbf{s}, \mathbf{c} ; \mathbf{x}) k(\mathbf{c}) g(\mathbf{c}) \mathrm{d} \mathbf{s} \mathrm{d} \mathbf{c} \\
=\int_{\Omega} \int_{\Theta} q(\mathbf{s}, \mathbf{c} ; \mathbf{x}) f(\mathbf{s}, \mathbf{c}) \mathrm{d} \mathbf{s} \mathrm{d} \mathbf{c}
\end{gathered}
$$

in which

$$
q(\mathbf{s}, \mathbf{c} ; \mathbf{x})=\int_{\Theta} Q(\mathbf{x}, \boldsymbol{\alpha},(D(w(\mathbf{s}, \mathbf{c} ; \boldsymbol{\alpha}, \mathbf{x})))) \mathrm{d} \boldsymbol{\alpha} .
$$

Corollary 10 shows that if $k(\boldsymbol{\theta}, \mathbf{x})$ and $Q(\mathbf{x}, \mathbf{s}, D(v(\mathbf{s}, \mathbf{x})))$ are independent of the variable $\boldsymbol{\theta}$, solving the IDE is converted to solving the integral equation (35) which only involves the variable $\mathbf{x}$. Hence, in this case, the problem is reduced by using Corollary 10.

\section{Examples}

In this section we present two simple examples. These examples are considered to illustrate the SMW formula for the IDE.

Example 1. We consider the following linear IDE firstly:

$$
\begin{gathered}
\partial_{x x} v(\theta, x)+\int_{0}^{1} e^{-\theta} \partial_{x x} v(\theta, x) \mathrm{d} \theta \\
=\pi^{2} \sin (\pi x)\left(e^{\theta}+1\right), \\
v(\theta, 0)=v(\theta, 1)=0 .
\end{gathered}
$$

According to Theorem 4, the solution of (37) can be written as

$$
v(\theta, x)=u(\theta, x)-p(\theta, x),
$$


where $u(\theta, x)$ satisfies

$$
\begin{gathered}
\partial_{x x} u(\theta, x)=\pi^{2} \sin (\pi x)\left(e^{\theta}+1\right), \\
u(\theta, 0)=u(\theta, 1)=0,
\end{gathered}
$$

$p(\theta, x)$ satisfies

$$
\begin{aligned}
\partial_{x x} p(\theta, x) & =g(x), \\
p(\theta, 0) & =p(\theta, 1)=0,
\end{aligned}
$$

and $g(x)$ satisfies

$$
g(x)+\int_{0}^{1} q(c, x) g(c) \mathrm{d} c=\int_{0}^{1} e^{-\theta} \partial_{x x} u(\theta, x) \mathrm{d} \theta
$$

in which

$$
\begin{aligned}
q(c, x) & =\int_{0}^{1} e^{-\theta} \partial_{x x} w(c, \theta, x) \mathrm{d} \theta=\int_{0}^{1} e^{-\theta} \delta(x-c) \mathrm{d} \theta \\
& =\delta(x-c)\left(1-e^{-1}\right)
\end{aligned}
$$

Solving (39) gives

$$
u(\theta, x)=-\sin (\pi x)\left(e^{\theta}+1\right) .
$$

Substituting (42) into (41) yields

$$
g(x)+\left(1-e^{-1}\right) g(x)=\pi^{2} \sin (\pi x)\left(2-e^{-1}\right) .
$$

In terms of (44), we have

$$
g(x)=\pi^{2} \sin (\pi x) .
$$

Once $g(x)$ is obtained, (40) can be solved:

$$
p(\theta, x)=-\sin (\pi x) \text {. }
$$

So the exact solution is

$$
\begin{aligned}
v(\theta, x) & =u(\theta, x)-p(\theta, x) \\
& =-\sin (\pi x) e^{\theta}-\sin (\pi x)+\sin (\pi x) \\
& =-\sin (\pi x) e^{\theta} .
\end{aligned}
$$

Example 2. Consider the following linear IDE:

$$
\begin{aligned}
\dot{v}(t)+\int_{0}^{\pi} \cos (t+s) v(s) \mathrm{d} s & =1, \\
v(0) & =0 .
\end{aligned}
$$

In terms of Corollary 9, (48) can be converted to the following equations:

$$
\begin{aligned}
& \dot{v}(t)=h(t), \\
& v(0)=0,
\end{aligned}
$$

where $h(t)$ satisfies

$$
h(t)+\int_{0}^{+\infty} q(\tau ; t) h(\tau) \mathrm{d} \tau=1
$$

in which

$$
\begin{aligned}
q(\tau ; t) & =\int_{0}^{\pi} \cos (t+s) w(\tau ; s) \mathrm{d} s \\
& = \begin{cases}\sin (t+\pi)-\sin (t+\tau), & \tau \leq \pi, \\
0, & \tau>\pi\end{cases}
\end{aligned}
$$

Substituting (51) into (50) yields

$$
\begin{gathered}
h(t)-\sin (t) \int_{0}^{\pi} h(\tau) \mathrm{d} \tau-\sin (t) \int_{0}^{\pi} \cos (\tau) h(\tau) \mathrm{d} \tau \\
\quad-\cos (t) \int_{0}^{\pi} \sin (\tau) h(\tau) \mathrm{d} \tau=1 .
\end{gathered}
$$

Solving (52) yields

$$
h(t)=1+\sin (t) \frac{-8 \pi}{4+\pi^{2}}+\cos (t) \frac{8-2 \pi^{2}}{4+\pi^{2}} .
$$

Combining (53) with (49) yields

$$
v(t)=t+\frac{8 \pi}{4+\pi^{2}}[\cos (t)-1]+\frac{8-2 \pi^{2}}{4+\pi^{2}} \sin (t)
$$

which is the exact solution.

\section{Conclusion}

In this work, we extend the SMW formula for the linear IDE. By using the SMW formula, the linear IDE can be decomposed into several linear differential equations and one pure integral equation. The SMW formula could be a powerful tool to solve the linear IDE. Combining the SMW formula with some analytical methods developed for the integral equation may provide the analytical approach for the linear IDE. The SMW formula can also be combined with the numerical methods for solving the linear IDE numerically.

\section{Competing Interests}

The author declares that there is no conflict of interests regarding the publication of this paper.

\section{Acknowledgments}

The author is grateful for the financial support of the Natural Science Foundation of China (nos. 11472076 and 51609034) and China Postdoctoral Science Foundation (no. 2016M590219).

\section{References}

[1] M. L. Lekala, G. J. Rampho, S. A. Sofianos, and R. M. Adam, "An integro-differential equation for Bose-Einstein condensates," Few-Body Systems, vol. 50, no. 1-4, pp. 427-429, 2011. 
[2] C. M. Elliott and S. McKee, "On the numerical solution of an integro-differential equation arising from wave-power hydraulics," BIT Numerical Mathematics, vol. 21, no. 3, pp. 317-325, 1981.

[3] M. Kostoglou, "Theoretical analysis of the warm-up of monolithic reactors under non-reacting conditions," Chemical Engineering Science, vol. 54, no. 17, pp. 3943-3953, 1999.

[4] G. Guerra and W. Shen, "Existence and stability of traveling waves for an integro-differential equation for slow erosion," Journal of Differential Equations, vol. 256, no. 1, pp. 253-282, 2014.

[5] T.-L. Bo, L. Xie, and X. J. Zheng, "Numerical approach to wind ripple in desert," International Journal of Nonlinear Sciences and Numerical Simulation, vol. 8, no. 2, pp. 223-228, 2007.

[6] C. M. Chen and T. M. Shih, Finite Element Methods for Integrodifferential Equations, World Scientific, Singaore, 1998.

[7] C. M. Huang, "Stability of linear multistep methods for delay integro-differential equations," Computers \& Mathematics with Applications, vol. 55, no. 12, pp. 2830-2838, 2008.

[8] M. Turkyilmazoglu, "An effective approach for numerical solutions of high-order Fredholm integro-differential equations," Applied Mathematics and Computation, vol. 227, pp. 384-398, 2014.

[9] A. Borhanifar and K. Sadri, "A new operational approach for numerical solution of generalized functional integro-differential equations," Journal of Computational and Applied Mathematics, vol. 279, pp. 80-96, 2015.

[10] H. Aminikhah, "A new analytical method for solving systems of linear integro-differential equations," Journal of King Saud University-Science, vol. 23, no. 4, pp. 349-353, 2011.

[11] M. Kostoglou, "On the analytical separation of variables solution for a class of partial integro-differential equations," Applied Mathematics Letters, vol. 18, no. 6, pp. 707-712, 2005.

[12] E. Hernández and J. P. C. Dos Santos, "Asymptotically almost periodic and almost periodic solutions for a class of partial integrodifferential equations," Electronic Journal of Differential Equations, vol. 38, pp. 1-8, 2006.

[13] W. H. Press, S. A. Teukolsky, W. T. Vetterling, and B. P. Flannery, Numerical Recipes in C: The Art of Scientific Computing, Cambridge University Press, Cambridge, UK, 2002. 


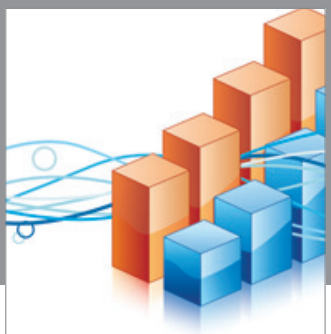

Advances in

Operations Research

vatem alat4

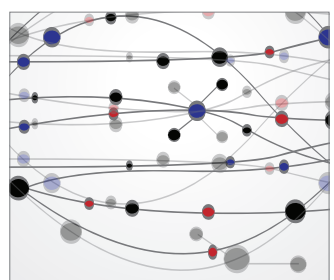

\section{The Scientific} World Journal
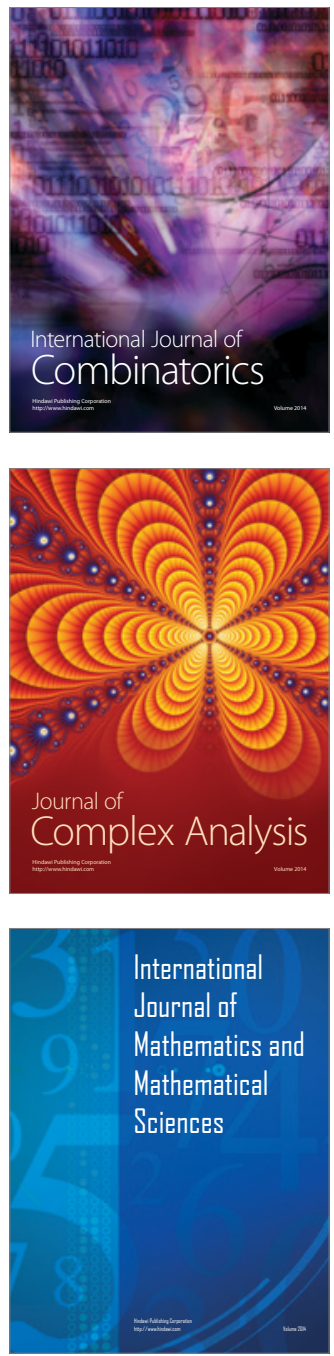
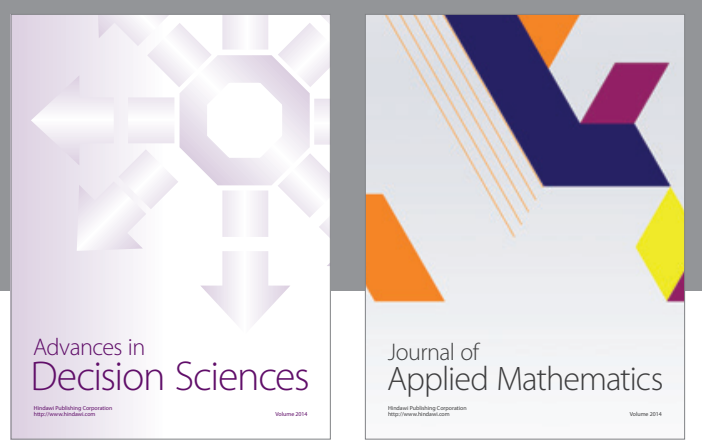

Algebra

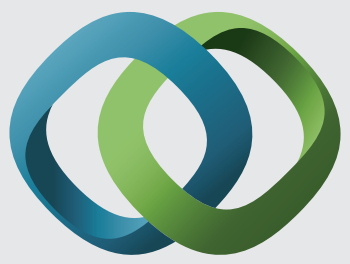

\section{Hindawi}

Submit your manuscripts at

http://www.hindawi.com
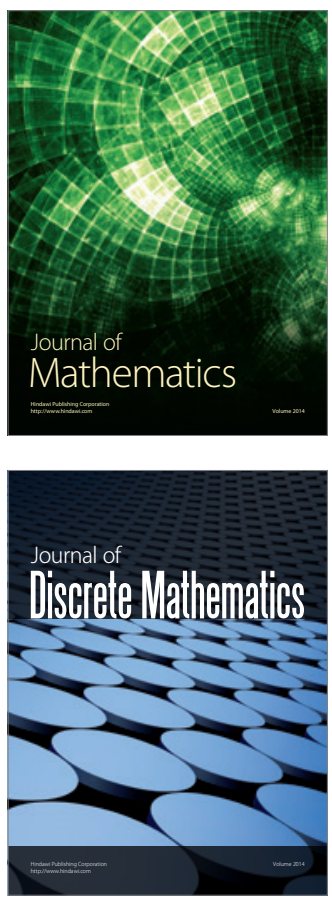

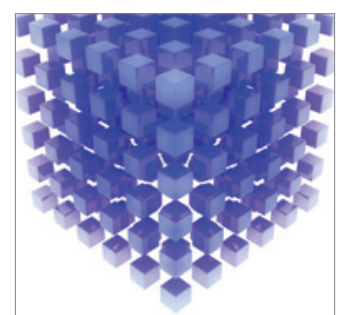

Mathematical Problems in Engineering
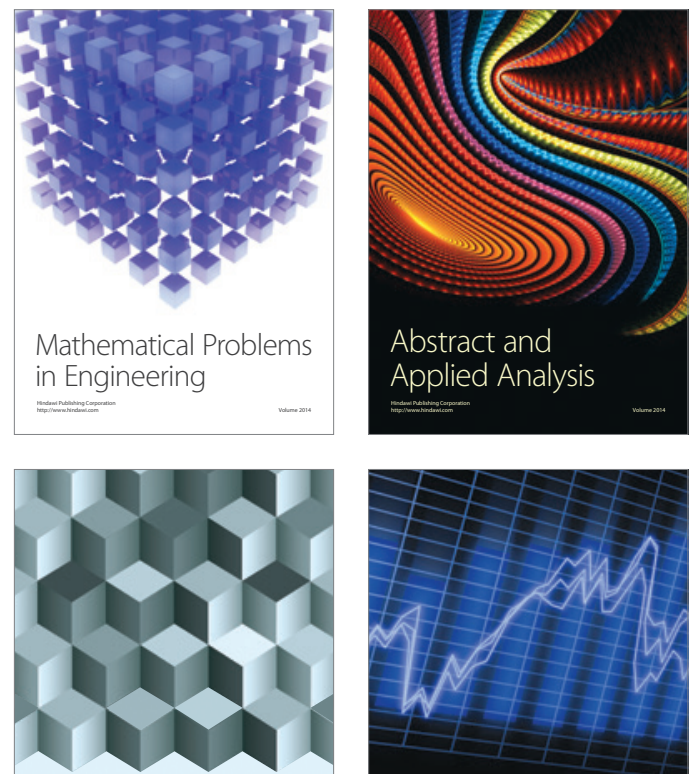

Journal of

Function Spaces

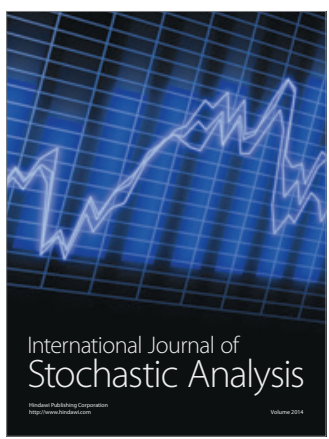

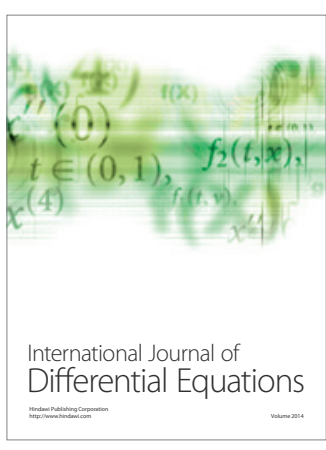
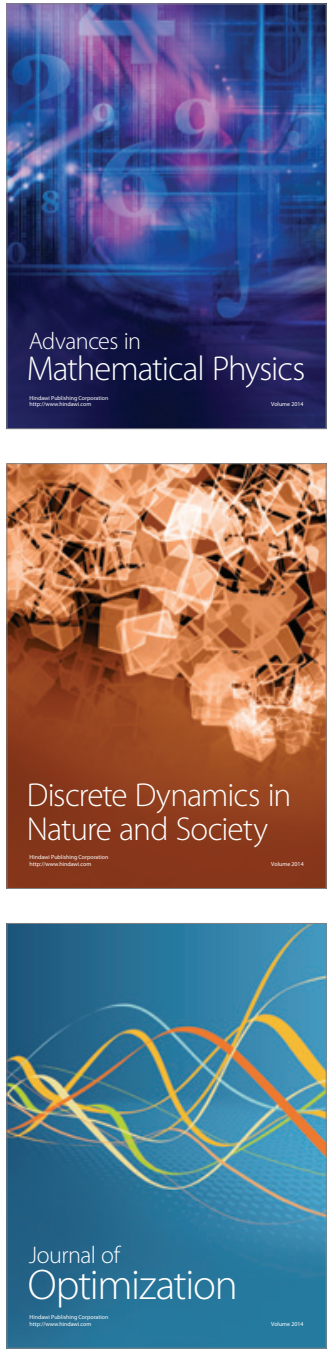